\title{
SHORTEST PERIODIC BILLIARD TRAJECTORIES IN CONVEX BODIES
}

\author{
MOHAMMAD GHOMI
}

\begin{abstract}
We show that the length of any periodic billiard trajectory in any convex body $K \subset \mathbf{R}^{n}$ is always at least 4 times the inradius of $\mathrm{K}$; the equality holds precisely when the width of $K$ is twice its inradius, e.g., $K$ is centrally symmetric, in which case we prove that the shortest periodic trajectories are all bouncing ball (2-link) orbits.
\end{abstract}

\section{INTRODUCTION}

Motivated by applications to inverse spectral problems ("can one hear the shape of a drum?"), S. Zelditch [5] has recently raised the question of whether every shortest periodic billiard trajectory in a bi-axisymmetric smooth convex planar body is a bouncing ball (2-link) orbit. Our main result (Theorem 1.2 below), which holds for all convex bodies in Euclidean space $\mathbf{R}^{n}$, implies that the answer is yes.

By a convex body $K \subset \mathbf{R}^{n}$ we mean a compact convex subset with interior points. A periodic billiard trajectory $T$ in $K$ is a sequence of distinct boundary points $p_{i} \in \partial K, i \in \mathbf{Z} / N \mathbf{Z}, N \geq 2$, called links of $T$, such that, for every $i$,

$$
n_{i}:=\frac{p_{i}-p_{i-1}}{\left\|p_{i}-p_{i-1}\right\|}+\frac{p_{i}-p_{i+1}}{\left\|p_{i}-p_{i+1}\right\|}
$$

is an (outward) support vector of $K$ at $p_{i}$; that is

$$
\left\langle x-p_{i}, n_{i}\right\rangle \leq 0, \quad \text { for all } x \in K .
$$

When $T$ has exactly two links $(N=2)$ we say it is a bouncing ball orbit. The length of $T$ is defined by

$$
\operatorname{length}(T):=\sum_{i=1}^{N}\left\|p_{i}-p_{i+1}\right\| .
$$

The main question we are concerned with in this paper is:

Problem 1.1. In which convex bodies are the shortest periodic billiard trajectories bouncing ball orbits?

Date: November 2002; Last typeset:June 1, 2003.

1991 Mathematics Subject Classification. Primary 52A20; Secondary 58J50, 37.

Key words and phrases. Billiards, convex body, bouncing ball orbit, inradius, width.

Partially supported by NSF grant DMS-0204190. 
The following result provides a sufficient criterion in terms of natural geometric measures: the inradius of $K$, which is the radius of the largest ball contained in $K$; and the width of $K$, which is the thickness of the narrowest slab which contains $K$.

Theorem 1.2. Let $K \subset \mathbf{R}^{n}$ be a convex body, and $T$ be a periodic billiard trajectory in $K$. Then

$$
\operatorname{length}(T) \geq 4 \operatorname{inradius}(K) \text {. }
$$

Further, the equality holds for some $T$, if and only if $\operatorname{width}(K)=2 \operatorname{inradius}(K)$. In this case, every shortest periodic trajectory of $K$ is a bouncing ball orbit.

We say $K$ is centrally symmetric provided that (after a translation) $K$ coincides with its reflection through the origin of $\mathbf{R}^{n}$ (this notion is weaker than "biaxisymmetric"). The above theorem yields:

Corollary 1.3. If $K \subset \mathbf{R}^{n}$ is a centrally symmetric convex body, then each of the shortest periodic billiard trajectories in $K$ is a bouncing ball orbit.

Proof. By theorem 1.2 it is enough to check that width $(K)=2 \operatorname{inradius}(K)$. Set $r:=\operatorname{inradius}(K)$. Then $K$ contains a ball $B$ of radius $r$. Let $B^{\prime}$ be the "central symmetrization" of $B$, that is,

$$
B^{\prime}:=\frac{B+(-B)}{2}=\left\{\frac{x-y}{2} \mid x, y \in B\right\} .
$$

Then $B^{\prime}$ is a ball of radius $r$ centered at the origin of $\mathbf{R}^{n}$. Further, since $K$ is centrally symmetric $(K=-K)$, it follows that $B^{\prime} \subset K$. Since the inradius of $K$ is $r$, the boundary of $K, \partial K$, must intersect $B^{\prime}$ at a point $p$. Then $-p$ also has to lie in $\partial K \cap B^{\prime}$ by symmetry. Let $H \subset \mathbf{R}^{n}$ be a support hyperplane of $K$ at $p$. Then $H$ is also a support hyperplane of $B^{\prime}$ which yields that $H$ is orthogonal to the segment $(-p) p$. By symmetry, the reflection of $H$, which we denote by $-H$, is also a supporting hyperplane, and is orthogonal to $(-p) p$ at $-p$. So the distance between $H$ and $-H$ is $2 r$, which yields that width $(K) \leq 2 r$. On the other hand, we always have $\operatorname{width}(K) \geq 2$ inradius $(K)=2 r$, which completes the proof.

Note 1.4. Without the central symmetry assumption, Corollary 1.3 does not in general remain valid. An equilateral triangle, whose corners may be rounded off by a small amount, would be a counterexample ${ }^{1}$. Here the shortest orbit is the (orthic) triangle which is obtained by connecting the midpoints of the sides (Figure 1(a)).

Note 1.5. As is known, and easy to show, any smooth convex planar body has at least two bouncing ball orbits (one is determined by the "diameter" and the other by the "width"). If one forgoes convexity, however, one may construct smooth planar regions without any bouncing ball orbits that are contained in

\footnotetext{
${ }^{1}$ This observation has been known to Yves Colin de Verdiere [6].
} 
the region (we say that a billiard trajectory is contained in a region if the line segment connecting any pairs of consecutive links is contained in that region). An example of a smooth region which does not contain any bouncing ball orbits is illustrated in Figure 1(b). This region has been known to Bos [1].

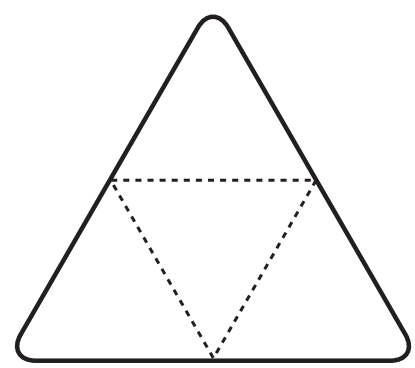

(a)

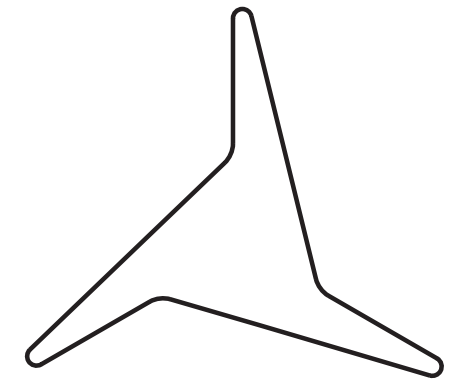

(b)

\section{FiguRE 1}

Note 1.6. Theorem 1.2 gives a lower bound for the length of shortest periodic trajectories of a convex body; however, this bound is sharp only when 2 inradius $(K)$ is equal to width $(K)$. Can one find a lower bound which is sharp in all cases? (Of course this lower bound should coincide with $4 \operatorname{inradius}(K)$ whenever 2 inradius $(K)$ is equal to $\operatorname{width}(K)$.) Can one find a sharp upper bound for the length of shortest periodic billiard trajectories of a convex body? One estimate, in terms of volume, is given by Viterbo [4].

\section{Proof of Theorem 1.2}

The proof of Theorem 1.2 follows from the two propositions below. By a polygon $P$ we mean a sequence of distinct points $p_{i} \in \mathbf{R}^{n}, i \in \mathbf{Z} / N \mathbf{Z}$ (in particular, every periodic billiard trajectory is a polygon). Each $p_{i}$ is called a vertex of $P$, and the length of $P$ is defined as $\sum_{i=1}^{N}\left\|p_{i}-p_{i+1}\right\|$. The convex hull of a set $X \subset \mathbf{R}^{n}$, which we denote by conv $X$, is the intersection of all convex sets which contain $X$.

Proposition 2.1. Let $B \subset \mathbf{R}^{n}$ be a ball of radius $r$, and $P \subset \mathbf{R}^{n}$ be a polygon such that the center of $B$ lies in conv $P$, and the vertices of $P$ lie outside the interior of $B$. Then length $(P) \geq 4 r$, with equality only if $P$ has exactly two vertices.

The proof of the above proposition follows from a little trigonometry (Lemma 3.1) together with some integral geometry (Crofton's formula). The proof of the following proposition, uses the above proposition and some basic convexity theory.

Proposition 2.2. Let $K \subset \mathbf{R}^{n}$ be a convex body, and $T$ be a periodic billiard trajectory in $K$. Suppose that there exists a ball $B$ of radius $r$ in $K$ whose center 
does not lie in conv $T$. Then length $(T) \geq 4 r$, with equality only if $T$ is a bouncing ball orbit.

The proof of the above propositions appear in the next two sections. Using these, we prove Theorem 1.2 as follows:

Let $r:=\operatorname{inradius}(K)$ and $B \subset K$ be a ball of radius $r$. Then either conv $T$ contains the center of $B$ or not. Thus Proposition 2.1 or Proposition 2.2 respectively establish that length $(T) \geq 4 r$.

Now suppose that length $(T)=4 r$, then, either by Proposition 2.1 or $2.2, T$ is a bouncing ball orbit. In particular, the support hyperplanes of $K$ which pass through each link of $T$, and are orthogonal to the support vectors $n_{i}$ given by (1), are parallel. So width $(K) \leq 2 r$. But we always have that width $(K) \geq 2 r$. So width $(K)=2 r$.

Conversely, suppose width $(K)=2 r$. Then there exists a ball $B$ of radius $r$ in $K$, and $K$ is contained in between a pair of parallel support hyperplanes of separation $2 r$. So $B$ must intersect both support hyperplanes, and it is clear that the intersection points generate a bouncing ball orbit $T$ with length $(T)=4 r$, since the line connecting the intersection points is orthogonal to both support hyperplanes.

So we conclude that width $(K)=2 r$ if and only if $K$ contains some bouncing ball orbit of length $4 r$. In particular, whenever width $(K)=2 r$, every shortest periodic trajectory $T \subset K$ has length $4 r$. Thus, since inradius $(K)=r$, it follows from Propositions 2.1 and 2.2, that every shortest periodic trajectory of $K$ is a bouncing ball orbit.

\section{Proof of Proposition 2.1}

Lemma 3.1. Let $0 \leq \alpha_{i} \leq \pi / 2, i=1, \ldots N$, and $\sum_{i=1}^{N} \alpha_{i} \geq \pi$. Then

$$
\sum_{i=1}^{N} \sin \alpha_{i} \geq 2,
$$

with equality if and only if all $\alpha_{i}=0$ save two.

Proof. Since $2 / \pi<1$, comparing the graph of $y=\sin x$ with that of $y=(2 / \pi) x$ quickly yields that

$$
\sin x \geq \frac{2}{\pi} x
$$

for all $0 \leq x \leq \pi / 2$. Thus, since by assumption $\sum_{i=1}^{N} \alpha_{i} \geq \pi$, we have

$$
\sum_{i=1}^{N} \sin \alpha_{i} \geq \frac{2}{\pi} \sum_{i=1}^{N} \alpha_{i} \geq 2 .
$$

If $\sum_{i=1}^{N} \sin \alpha_{i}=2$, then, the above expression yields

$$
\sum_{i=1}^{N} \sin \alpha_{i}=\frac{2}{\pi} \sum_{i=1}^{N} \alpha_{i} .
$$


But $\sin \alpha_{i} \geq(2 / \pi) \alpha_{i}$, thus the above equality yields

$$
\sin \alpha_{i}=\frac{2}{\pi} \alpha_{i}
$$

The last equality holds only when $\alpha_{i}=0$, or $\alpha_{i}=\pi / 2$. Thus we conclude that the equality holds in (3) precisely when all $\alpha_{i}=0$ save two.

Let $p_{i}, i=1, \ldots, N$, be the vertices of $P, o$ be the center of the ball $B$, which we may assume to coincide with the origin of $\mathbf{R}^{n}$, and $2 \alpha_{i}:=\angle p_{i} o p_{i+1}$ be the corresponding angles. We claim that

$$
\sum_{i=1}^{N} \alpha_{i} \geq \pi .
$$

To see this note that $\sum_{i=1}^{N} 2 \alpha_{i}$ is equal to the length of the projection of $P$, say $P^{\prime}$, into a sphere $S$ of radius 1 centered at $o$. Since $o$ is in the convex hull of $P$, o lies in the convex hull of $P^{\prime}$ as well. In particular, every great circle in $S$ intersects $P^{\prime}$; thus, it follows from Crofton's formula [2] that length $\left(P^{\prime}\right) \geq 2 \pi$. So (4) holds which in turn allows us to apply Lemma 3.1 to conclude that (3) holds as well.

Next note that, since by assumption $\left\|p_{i}\right\| \geq r$,

$$
\begin{aligned}
\left\|p_{i}-p_{i+1}\right\|^{2} & =\left\|p_{i}\right\|^{2}+\left\|p_{i+1}\right\|^{2}-2\left\|p_{i}\right\|\left\|p_{i+1}\right\| \cos 2 \alpha_{i} \\
& \geq 2 r^{2}\left(1-\cos 2 \alpha_{i}\right) \\
& =4 r^{2} \sin ^{2} \alpha_{i} .
\end{aligned}
$$

Thus, the above inequality together with (3) yield that

$$
\begin{aligned}
\operatorname{length}(P) & \geq \sum_{i=1}^{N}\left\|p_{i}-p_{i+1}\right\| \\
& \geq 2 r \sum_{i=1}^{N} \sin \alpha_{i} \\
& \geq 4 r .
\end{aligned}
$$

Now suppose that the equality in the above inequality holds. Then, by Lemma 3.1, all $\alpha_{i}=0$, save two. In this case the remaining two angles must each be equal to $\pi / 2$ by (4). Thus all vertices of $P$ must lie on a line which passes through the center $o$ of $B$. Since the vertices of $P$ lie outside of the interior of $B$, and are distinct, it then follows that $P$ has exactly two vertices.

\section{Proof of Proposition 2.2}

Since, by assumption, the center $o$ of $B$ does not lie in conv $T$, which is a convex set, there exists a unique point $p$ of $\operatorname{conv} T$ which is closest to $o$ [3]. After a rigid 
motion we may assume that $o$ is the origin of $\mathbf{R}^{n}$, and $p$ lies on the negative half of the $x_{n}$-axis. So

$$
\frac{p}{\|p\|}=(0,0, \ldots,-1)
$$

and $p$ is the "highest" point of conv $T$. That is,

$$
\pi(p) \geq \pi\left(p_{i}\right)
$$

where $\pi: \mathbf{R}^{n} \rightarrow \mathbf{R}$ is projection into the $n^{t h}$ coordinate and $p_{i}, i=1, \ldots, N$ are the links of $T$. Suppose, towards a contradiction, that $p \in T$. That is $p=p_{k}$, a link of $T$. Let $n:=n_{k}$ be as in (1). That is,

$$
n:=\frac{p-p_{k-1}}{\left\|p-p_{k-1}\right\|}+\frac{p-p_{k+1}}{\left\|p-p_{k+1}\right\|} .
$$

Combining the previous two displayed expressions yields

$$
\pi(n) \geq 0 \text {. }
$$

On the other hand, since $o$ is an interior point of $K$ and $n$ is a support vector, (5) and (2) yields that

$$
\pi(n)=\left\langle-\frac{p}{\|p\|}, n\right\rangle=\frac{1}{\|p\|}\langle o-p, n\rangle<0,
$$

which is a contradiction. So we conclude that $p \notin T$.

Since $p \in \partial \operatorname{conv} T$, but $p \notin T$, it follows that $p$ lies in the (relative) interior of a face $F$ of $T$. Let $p_{k}$ be a vertex of $F$. Note that since no link of $T$ is higher than $p, F$ has to be "horizontal" (parallel to $x_{n}=0$ hyperplane), which yields that

$$
\pi\left(p_{k}\right)=\pi(p) \geq \pi\left(p_{i}\right)
$$

for all $i$. So, as we had argued earlier, since

$$
n_{k}:=\frac{p_{k}-p_{k-1}}{\left\|p_{k}-p_{k-1}\right\|}+\frac{p_{k}-p_{k+1}}{\left\|p_{k}-p_{k+1}\right\|},
$$

it follows that

$$
\pi\left(n_{k}\right) \geq 0
$$

which in turn yields

$$
\left\langle p, n_{k}\right\rangle=\|p\|\left\langle\frac{p}{\|p\|}, n_{k}\right\rangle=-\|p\| \pi\left(n_{k}\right) \leq 0 .
$$

Further, note that, since $o \in K,(2)$ yields

$$
\left\langle p_{k}, n_{k}\right\rangle=-\left\langle o-p_{k}, n_{k}\right\rangle \geq 0 \text {. }
$$

The previous two inequalities yield:

$$
\left\langle p_{k}-p, n_{k}\right\rangle=\left\langle p_{k}, n_{k}\right\rangle-\left\langle p, n_{k}\right\rangle \geq\left\langle p_{k}, n_{k}\right\rangle .
$$

Next, recall that, since $p \notin T, p \neq p_{k}$. Thus

$$
r \frac{p_{k}-p}{\left\|p_{k}-p\right\|} \in B \subset K
$$


This via (2) yields

which we may rewrite as

$$
\left\langle r \frac{p_{k}-p}{\left\|p_{k}-p\right\|}-p_{k}, n_{k}\right\rangle \leq 0,
$$

$$
r\left\langle p_{k}-p, n_{k}\right\rangle \leq\left\|p_{k}-p\right\|\left\langle p_{k}, n_{k}\right\rangle .
$$

Comparing the above inequality with (6), we obtain

$$
r \leq\left\|p_{k}-p\right\|
$$

Thus all the vertices of the face $F$ are at least a distance $r$ away from $p$. Let $T_{F}$ be the subpolygon of $T$ composed of the vertices of $F$, and with the ordering inherited from $T$, then the triangle inequality together with Proposition 2.1 yield

$$
\operatorname{length}(T) \geq \operatorname{length}\left(T_{F}\right) \geq 2 r .
$$

If length $(T)=2 r$, then the above expression yields that length $\left(T_{F}\right)=\operatorname{length}(T)$, which implies $T_{F}=T$. Further, we also get length $\left(T_{F}\right)=2 r$, which, by Proposition 2.1, implies that $T_{F}$ has only two vertices, which completes the proof.

\section{ACKNOWLEDGEMENT}

The author thanks Steve Zelditch for bringing Problem 1.1 to the author's attention, Serge Tabachnikov for his careful reading of an early draft of this note, Kate Hurley for suggesting a convenient proof for Lemma 3.1, and the referee for

pointing out that the example depicted in Figure 1(b) had been known earlier to Bos .

\section{REFERENCES}

[1] W. Bos, Kritische Sehnen auf Riemannschen Elementarraumstücken, Math. Ann. 151 (1963), 431-451.

[2] S. S. Chern, Curves and surfaces in Euclidean space, in Studies in Global Geometry and Analysis, pages 16-56. Math. Assoc. Amer., Prentice-Hall, Englewood Cliffs, N.J., 1967.

[3] R. Schneider, Convex Bodies: The Brunn-Minkowski Theory, Encyclopedia of mathematics and its applications, v. 44, Cambridge University Press, Cambridge, UK, 1993.

[4] C. Viterbo, Metric and isoperimetric problems in symplectic geometry, J. Amer. Math. Soc. 13 (2000), 411-431.

[5] S. Zelditch, Spectral determination of analytic bi-axisymmetric plane domains. Geom. Funct. Anal. 10 (2000), no. 3, 628-677.

[6] S. Zelditch, Personal communication, October 2002.

School of Mathematics, Georgia Institute of Technology, Atlanta, GA 30332

E-mail address: ghomi@math.gatech.edu

$U R L$ : www . math.gatech. edu/ ghomi 